\title{
Interoception and Emotion: Shared Mechanisms and Clinical Implications
}

\author{
Lisa Quadt ${ }^{1}$, Hugo D. Critchley ${ }^{1,2,3}$ and Sarah N Garfinkel ${ }^{1,2,3}$
}

${ }^{1}$ Psychiatry, Department of Neuroscience, Brighton and Sussex Medical School (BSMS), University of Sussex, Brighton, UK

${ }^{2}$ Sackler Centre for Consciousness Science, University of Sussex, Brighton, UK

${ }^{3}$ Sussex Partnership NHS Foundation Trust

\section{Abstract}

Internal states of bodily arousal contribute to emotional feeling states and behaviours. This review details the influence of interoceptive processing on emotion and describes how deficits in interoceptive ability may underpin aberrant emotional processes characteristic of clinical conditions. The representation and control of bodily physiology (e.g. heart rate and blood pressure) and the encoding of emotional experience and behaviour share neural substrates within forebrain regions coupled to ascending neuromodulatory systems. This functional architecture provides a basis for dynamic embodiment of emotion. We will approach the relationship between interoception and emotion within the interoceptive predictive processing framework and describe how emotional states could be the product of interoceptive prediction error minimization.

Keywords

Interoception; emotion; predictive processing; individual differences; autism; anxiety; insula 
1. Introduction

Emotions are often accompanied by bodily changes - we experience this when we blush with embarrassment, feel our heart beat faster and our breath go shallow when we are in fear. The view that emotion and body are intimately related was first formulated by William James (James, 1884) who claimed that peripheral autonomic changes constitute emotions. The sensing of autonomic changes is referred to as interoception, the afferent processing of signals that originate within the body. Involved in interoception are different classes and channels (e.g., cardiovascular, gastric) of information that are distinct with respect to their afferent pathway (neural, humoral) and the generation of the signal (mechanoreceptive organ stretching, chemoreception). These channels share neural substrates, i.e., brain mechanisms, in which integrative processes take place. These allow for the generation of representations that predict internal states, which might steer adaptive behaviour (e.g., when blood sugar levels drop below a specific threshold, find food). Changes in bodily states and their interoceptive signaling can be constitutive of emotional feelings, leading to the possibility that the affective style (e.g. the intensity of emotions) of a person reflects differences in conscious and unconscious processing of interoceptive information.

Individual interoceptive differences manifest themselves in different psychological dimensions of interoception that comprise objective, subjective, and metacognitive measures (Canales-Johnson et al., 2015; Garfinkel, Seth, Barrett, Suzuki, \& Critchley, 2015). Quantifications of interoceptive abilities include objective measures such as behavioural performance accuracy during a heartbeat detection tasks (Schandry, 1981). Subjective (i.e. sensibility) measures include self-reports about interoceptive proficiency, such as evaluations of how well internal signals can be sensed using questionnaires (Porges, 1993). An enhanced mismatch between subjective and objective measures of interoception is associated with anxiety symptomatology (Garfinkel et al., 2016).

A way in which bodily and emotional processes merge can be found within the brain, where they share a similar neural architecture (Zaki, Davis, \& Ochsner, 2012). Specific cortical regions, especially the anterior insular cortex (AIC), are activated when individuals focus their attention on changes in internal, bodily states, highlighting the role of AIC in the perception of autonomic changes (Critchley, 2004; Peyron, Laurent, \& Garcia-Larrea, 2000; Williams et al., 2000). The insular cortex is thus of special interest to both interoception and emotion. In the brain, fibers from lamina 1 project into the NTS (nucleus of the solitary tract), parabrachial nucleus, periaqueductal gray, and other brainstem autonomic output nuclei. However, the ventromedial posterior nucleus of the thalamus is the main relay of viscerosensory information within the spinothalamic tract projecting onto insular cortex (see Critchley and Harrison, 2013). The insular cortex is located in the brain bilaterally underneath frontal and temporal lobes and is part of the cerebral cortex. It is divided into 
posterior and anterior insular cortex, where each part has its own cellular and functional structure. Its cytoarchitecture changes from granular in the posterior region to agranular in the anterior insula. The insular cortex is bidirectionally connected to parietal, frontal and limbic regions (Deen, Pitskel, \& Pelphrey, 2011). While the posterior regions of the insula are reciprocally connected with the second somatosensory cortex (SII) and receive input from the thalamus, conveying information such as pain, temperature and oxygen status, AIC is closely connected with the anterior cingulate cortex (ACC). AIC and ACC together engage subcortical regions such as the amygdala and orbitofrontal cortex (OFC). It has thus been suggested that the posterior parts of the insula support primary, objective representations of bodily signals, while AIC re-represents and integrates these signals with exteroceptive and motivational information (Seth, 2013a). AIC is also engaged in emotion elicitation tasks (Kober et al., 2008; Wicker et al., 2003) and neuroscientific models posit that the processing of visceral information in AIC supports an overlap between interoceptive and emotional states (Lamm \& Singer, 2010).

Appraisal theories of emotion (Schachter \& Singer, 1962) add another component to the mix of interoception and emotion, namely that of cognitive appraisal. According to this view, emotional states are elicited by bodily changes and subsequent contextualization. Modern theories of the embodiment of emotion (Craig, 2002; Critchley, et al., 2004) adopt a similar approach, by also integrating findings about the shared neural underpinnings of interoception and emotion with the idea of contextualization. Together they highlight the role of bodily signals in shaping emotional experience and the role of cognitive appraisal in shaping the type of emotion felt.

These theoretical and empirical advances on the interplay of emotional and interoceptive processes have recently been put together within the unifying perspective of predictive processing (Clark, 2016). Predictive processing (PP) views the brain as a hierarchical generator of predictions about its most likely next states. These predictions are then compared against actual sensory input (generating a prediction error), which either leads to a change of predictions in light of the new evidence (perception), or to action that changes brain-external circumstances (active inference). This principle not only applies to exteroceptive information processing, but also to predicting and controlling bodily interoceptive states. This can be described as interoceptive inference (Seth, 2013), which has also been called 'interoceptive predictive processing' (IPP). IPP claims that emotional states are brought forth in the process of minimizing interoceptive prediction error and the top-down (i.e., from higher to lower levels in the processing hierarchy) predictive inference of causes of interoceptive signals. The network of AIC and other cortical regions is thought to underlie this process of generating emotions via interoceptive inference (Seth, Suzuki, \& Critchley, 2011). 
In this chapter, we will elaborate on the IPP perspective to frame findings about the relationship between emotion and interoception. We will detail shared mechanisms of emotional and interoceptive processing, focusing on the role of the insular cortex as a central hub in addition to core regions such as anterior cingulate cortex (ACC) and amygdala. In a next step, we describe how differences in interoceptive processing can lead to individual differences in emotional expression, behaviour, and experience. Lastly, we show how impairments in interoception relate to affective psychopathology, with particular focus on autism spectrum conditions (ASCs), anxiety disorders, depression, and eating disorders (EDs).

\section{Shared Mechanisms}

The relation between emotion and interoception is reflected in their neural counterparts. Previous findings support the description of a joint network of interoceptive and emotional processing that has AIC and ACC at its center. Imaging studies show that emotions are mediated by a matrix of subcortical and cortical structures whose activity is also correlated to alterations in internal bodily states (Phan, Wager, Taylor, \& Liberzon, 2002). Specifically, ventral prefrontal, anterior cingulate and insular cortices, amygdala, ventral striatum and dorsal brainstem are identified as relevant structures for emotions, which also have been related to changes in heart rate (Critchley et al., 2005), temperature (Nummenmaa, Glerean, Hari, \& Hietanen, 2014), and blood pressure (Critchley, Corfield, Chandler, Mathias, \& Dolan, 2000).

The insula and ACC play an especially important role for the joint processing of emotion and interoception. AIC is seen as mediating both interoceptive accuracy (i.e., how well individuals perceive inner states; Critchley et al. 2004) and deficits in emotion processing (Berthoz et al., 2002). This is in line with the claim that integration processes in the anterior insula allow the uncovering of internal bodily states which then inform emotional processing (Terasawa, Shibata, Moriguchi, \& Umeda, 2013). Thus there appears to be a triadic relationship between activity in AIC, emotional and interoceptive processing. Specifically, heightened activity in the insula is associated with both interoceptive processing and the processing of emotional intensity (Zaki, et al., 2012), supporting the view that judgements of emotional intensity are informed by the sensing of internal bodily sensations.

An instance of how interoceptive signaling influences emotion processing is how responses to emotional stimuli vary depending on when in the cardiac cycle they are presented (Critchley \& Garfinkel, 2017). Fear stimuli, for example, are more easily detected and perceived as more intense when presented at systole when baroreceptors (interoceptors that encode blood vessel and cardiac chamber stretch due to pressure change) are active compared to presentation at diastole when baroreceptors are inactive 
(Critchley \& Garfinkel, 2015; Garfinkel \& Critchley, 2016; Garfinkel et al., 2014). Cardiovascular arousal thus can enhance the experience of fear and anxiety.

Another example of the interacting relationship between interoception and emotion can be found in the influence of inflammation on emotional states. Inflammatory processes can lead to 'sickness behaviours' like fatigue, social withdrawal and irritability (Harrison, 2017). Interoceptive pathways to insula are involved in conveying inflammation signals, thus suggesting an interoceptive mechanism in the shared relation between inflammatory and emotional states.

These findings show that emotional and interoceptive processing have a common underlying neural architecture, which comprises AIC, ACC and other functionally connected regions. Bodily arousal and emotional intensity are tightly correlated, which points towards shared mechanisms beyond the brain.

\section{Individual Differences}

People differ with respect to how intensely they experience emotions in general, and it is particularly interesting that this might be due to individual differences in interoceptive performance. It is typically assumed that a heightened ability to detect internal bodily changes is associated with more intensely felt emotions, a hypothesis that has been confirmed in several studies (Wiens, Mezzacappa, \& Katkin, 2000).

Quantifying interoceptive performance involves a variety of methods, ranging from questionnaires to behavioural tests that look for fluctuations in physiological signals or experimentally alter organ physiology. Among the most widely used methods are heartbeat detection tasks, where individuals either count their heartbeats silently for a given amount of time (Schandry, 1981) or judge whether an external signal is in or out of synch with their heartbeat (Katkin, Morell, Goldband, Bernstein, \& Wise, 1982). In order to grasp these different measures, novel conceptual tools have been introduced to describe different psychological dimensions of interoception; viz. objective performance accuracy, subjective sensibility and metacognitive awareness (Garfinkel, et al., 2016).

Interoceptive accuracy refers to the objective measure of how well people perform on interoceptive tasks, such as heartbeat discrimination or counting tasks. This dimension is dissociable from sensibility, which refers to the subjective impression of how well individuals think they are able to perceive internal signals. This can be measured by questionnaires such as the Body Perception Questionnaire (Porges, 1993). The third dimension describes the relationship between subjective and objective measures, that is, the metacognitive awareness of individuals regarding how well they performed on an interoceptive task (e.g., knowing they performed well when they actually performed well or knowing they performed poorly when they did perform poorly), such as through confidence-accuracy 
correspondence. Thus metacognitive awareness can be conceptualized as an interoceptive insight measure. Deficits within these dimensions can predict emotional states and affective psychopathology. In line with this, individuals with higher alexithymia - a sub-clinical condition that is characterized by impairments in recognizing and describing one's emotions (Nemiah, 1976) - showed impoverished interoceptive accuracy (Ernst et al., 2014).

The three-level model of interoceptive dimensions was recently extended (Critchley \& Garfinkel, 2017) to include lower levels that exhibit afferent neural trafficking and the preconscious impact of interoceptive signals on sensory processing (Fig.1). Good heartbeat perceivers, for example, experience greater arousal for emotional pictures than poor heartbeat perceivers and also showed greater heartbeat-evoked potentials (HEP) for emotional pictures (Herbert, Pollatos, \& Schandry, 2007). Within brain, individual differences in insula activation correlate to levels of negative emotional states expressed as anxiety and neuroticism (Terasawa, et al., 2013). This again points into the direction of a link between interoceptive and emotional performance. Additionally, higher levels beyond metacognition are added to the model, which include executive processes like the modulation of attention (Critchley \& Garfinkel, 2017). Individual differences could manifest themselves in the ability to flexibly attend to either interoceptive or exteroceptive signals or switching between interoceptive tasks. This in turn may be related to focusing on and consciously detecting one's own emotional state.

The ability to detect emotion and its relationship to interoceptive performance may also extend into the social domain and influence how accurately emotions of others are perceived. Empirical results on this topic are, however, conflicting, possibly due to different measurement methods. On the one hand, it was found that the higher the interoceptive accuracy, the more sensitive one is to the emotions of another person (Terasawa, Moriguchi, Tochizawa, \& Umeda, 2014). In line with this finding is a study showing that people who scored high on alexithymia questionnaires showed reduced activation in insular cortex both when they tried to access their own emotions and when empathizing with another person's pain (Bird et al., 2010; Silani et al., 2008). Another indication for a close relationship between interoception and empathy is a finding that shows enhanced neural activity during empathy in brain regions related to both interoception and empathy after participants completed an interoceptive performance task (Ernst, Northoff, Böker, Seifritz, \& Grimm, 2013). The insula cortex underlies both current and predictive representations, which are crucial for learning processes of uncertainty and emotional states (Singer, Critchley, \& Preuschoff, 2009). In this learning process, predictions about feeling states in self and others are compared to actual incoming signals and then updated depending on the 
discrepancy between prediction and signal ${ }^{1}$. Integrative processes within the insula cortex support this error-based learning process in which empathic and subjective feeling states are generated.

\section{Dimensions of Interoception}

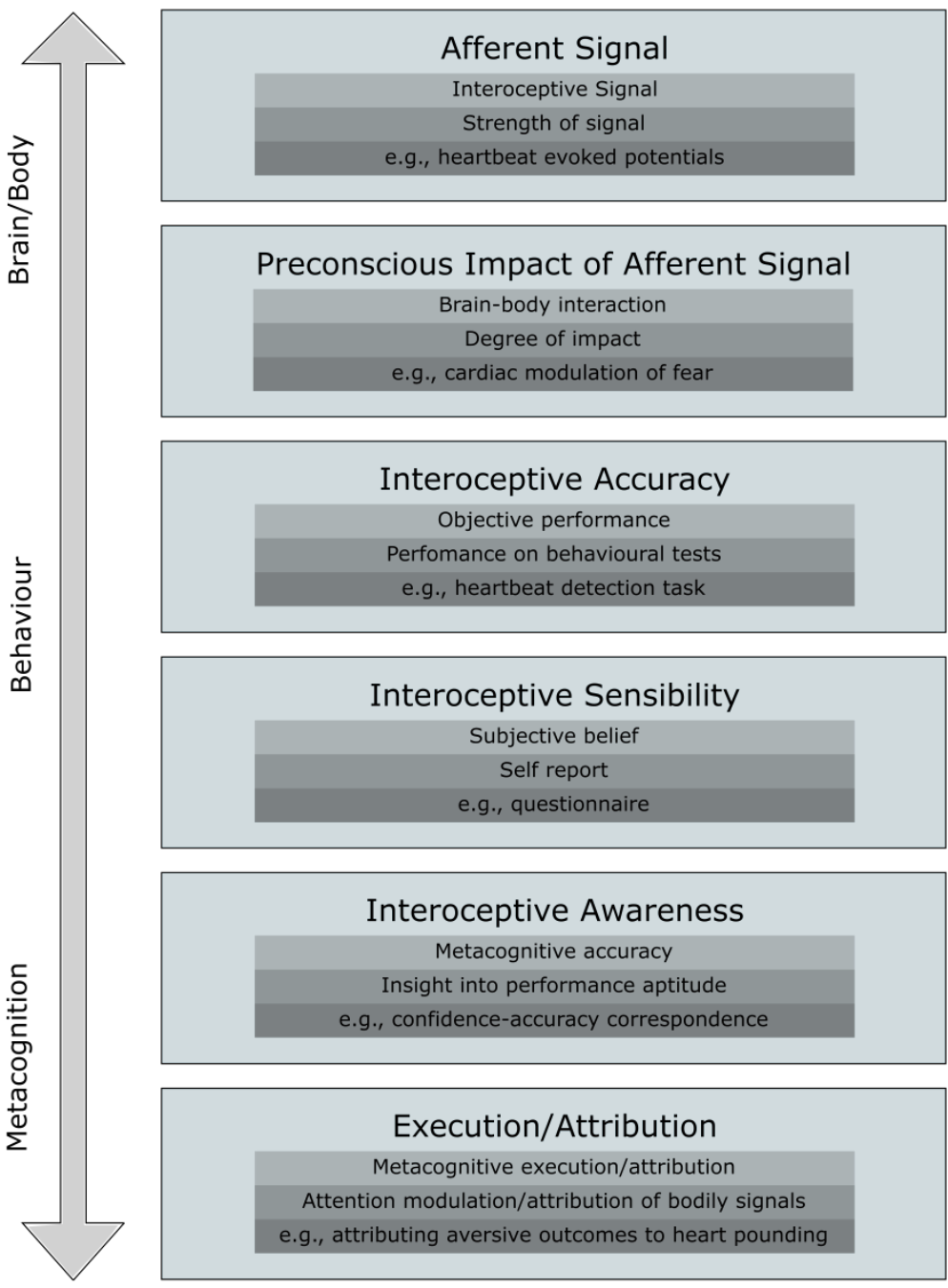

Figure 1 Dimensions of Interoception

Dimensions of interoception range from the level of brain and body, to behaviour, and metacognition. At each dimension, individual differences manifest themselves and can have an impact on emotional processes and states. These dimensions are though to interact with each other, for example in the alignment of interoceptive accuracy and sensibility.

\footnotetext{
${ }^{1}$ There might be confusion in the PP scheme about what is considered a 'signal' and what counts as a 'representation' (thanks to our reviewer for bringing up this important issue). A helpful distinction could be that representations exist even at very low levels, if representations are defined as abstractions from the actual signal. As soon as there is a prediction about what the signal (e.g., mechanoreceptive organ stretching) might be, there is an abstraction from the signal itself, thus making it a representation (for a more detailed discussion, see Quadt, 2017, chapter 6).
} 
On the other hand, Ainley and colleagues (Ainley, Maister, \& Tsakiris, 2015) did not find any correlations between interoceptive accuracy and empathy scores on questionnaires. The relationship between empathy and interoceptive performance thus is still up to debate.

In summary, these findings strongly suggest that the degree to which one is able to detect and attend to internal states determines how well emotions are recognized in self and others. These individual differences can be found along different dimensions of interoception, which interact with and influence emotional experience.

4. Interoceptive Predictive Processing (IPP) and Emotion

Predictive processing (PP;Clark, 2013, 2016) is ultimately a theory about how the brain makes sense of the world and body it is embedded in. While it has historically been exclusively applied to perception and action, it has recently been extended to include interoception and emotion (Seth et al., 2011).

\section{Predictive Processing}

The rationale of PP is that there are hidden causes in the world - hidden from the brain in the skull - that need to be inferred. All the brain can access are its own states: it can neither directly access the external world, nor the body it is embedded in, nor other agents. It thus has to solve an inverse problem, viz. how do the effects on the brain relate to causes in the external world (Hohwy, 2013). The solution to this problem presents itself in the form of inference; in order to find out about what lies behind the effects the brain receives, the causes must be inferred. It is proposed that this happens within a hierarchy where predictions about the most likely state of the level below are generated and then compared to actual sensory input. While low levels predict basic sensory properties of incoming signals at fast timescales, higher levels predict more complex regularities at slower timescales (Hohwy, 2010). The crucial information, i.e., the mismatch (i.e., error) between prediction and signal is then conveyed via feedforward connections in order to improve the model. This process of updating the model is called perceptual inference and is contrasted against active inference (Friston, 2010). In active inference, instead of changing models, biological agents act so to change sensory input to make it accommodate predictions better. Both perceptual and active inference serve the brain's main task: minimizing prediction error.

One question that arises, though, is the following: How does the brain 'decide' which of these strategies to choose? This is determined by how precise the expectations and error signals are deemed to be. The 'job' of prediction errors, as we have seen, is to improve the generative model. However, it is important that errors are not simply taken to be 'trustworthy' (i.e. reliably informing the system about external states) and change the model or motivate to act. Error signals are taken into account depending on an estimation of how reliable they will be. If prediction errors are, for example, estimated as highly precise, they are likely to 
change the hypothesis. That is, the impact of an error signal depends on how precise it is estimated to be. At the same time, the reliability of predictive models needs to factored in, too. Thus, "we are, in effect, estimating the uncertainty of our own representations of the world." (Clark, 2015, p. 12)

\section{Interoceptive Predictive Processing (IPP)}

This PP scheme was recently applied to interoception (Barrett \& Simmons, 2015; Seth, 2013b; Seth, et al., 2011). In general, interoceptive predictive processing (IPP) follows the same principle as inference of exteroceptive states as described above. Predictive models of the next most probable interoceptive signals are generated and compared to actual input. This mechanism serves to keep bodily states in their expected range and to flexibly adapt to external and internal changes. Instead of simply comparing inputs to homeostatic set-points and changing the internal environment accordingly, the idea is that the system anticipates demands that deviate from the average and thus efficiently regulates needs and resources. The terms 'allostasis' and 'predictive regulation' were introduced to conceptualize this process of optimizing regulation in a predictive manner (Sterling, 2012). According to the size and estimated reliability of the prediction error, prediction error can be minimized in several ways; namely by updating generative models; of interoceptive states; by engaging hormonal, visceral, immunological and autonomic mechanisms to change interoceptive signals via active inference; or by actions that change external conditions which have an impact on (Fig 2, (Seth, 2015)). 

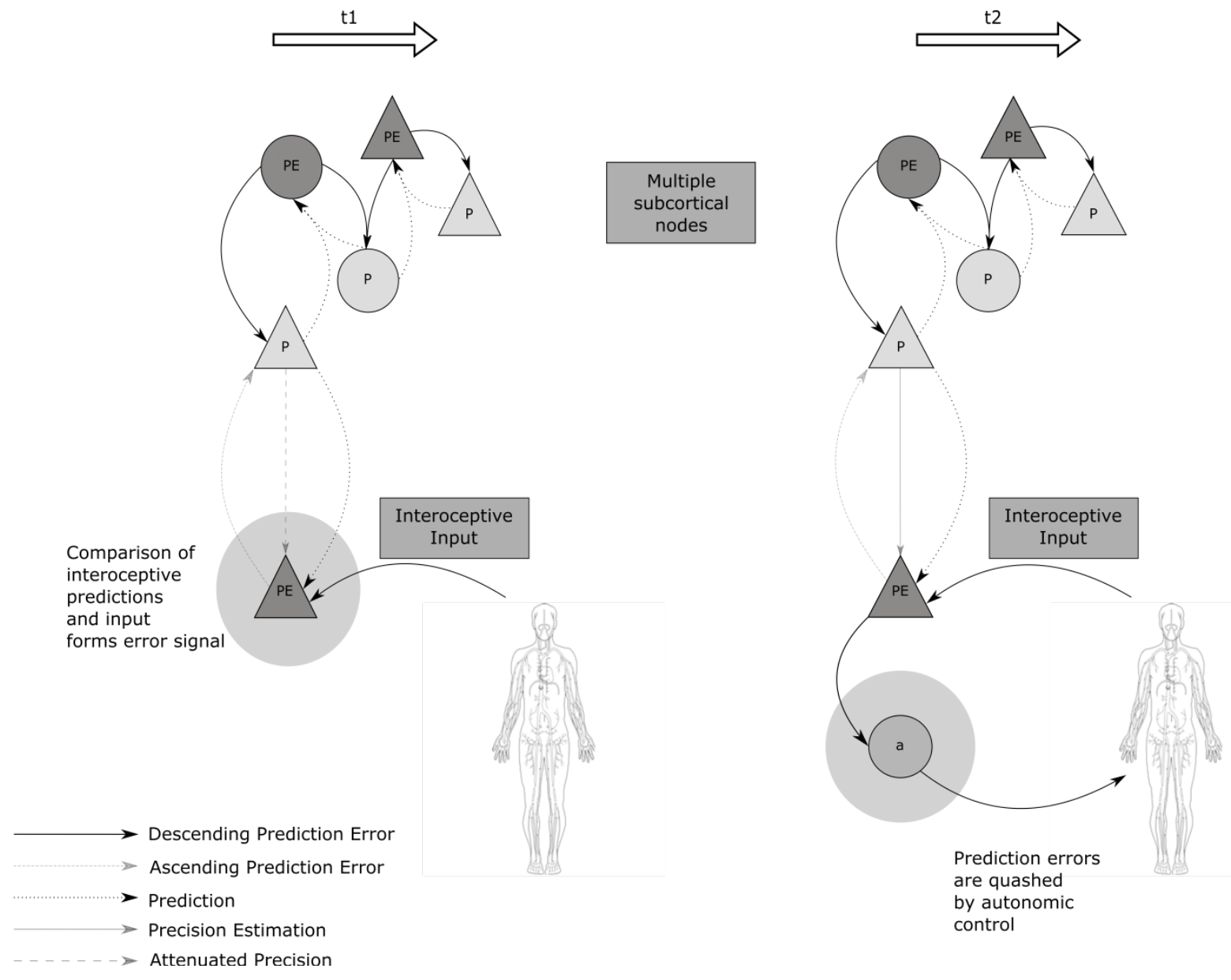

\section{Figure 2: Active interoceptive inference}

This figure depicts a sketch of the dynamics of active interoceptive inference. At time t1, predictions (' $\mathrm{P}$ ') about the interoceptive state of the body are passed down via backward connections and compared against actual incoming interoceptive input. When there is a mismatch between predicted and actual body state, a prediction error ('PE') is generated. Note that precision of this ascending error signal must be attenuated, so not to change predictions and thus to inhibit autonomic control. At time $\mathrm{t} 2$, prediction errors are quashed by autonomic control. The changed interoceptive input is again fed forward to now inform predictions.

The engagement of autonomic, hormonal, visceral, and immunological mechanisms serves to maintain homeostasis or to enable allostasis. ${ }^{2}$ Throughout the process of monitoring its own states, the system keeps updating and changing its states, in order to keep them in an expected range. Active interoceptive inference describes the process of adjusting how internal systems use autonomic, immunological and metabolic resources

\footnotetext{
${ }^{2}$ The difference between homeostasis and allostasis is that the former refers to the maintenance of specific and rather rigid set-points, while the latter describes a highly flexible demand-regulated change of states. Rather than committing to homeostatic set-points (e.g., keeping blood pressure around a specific value), allostasis anticipates the changing needs of the system and adjusts bodily states accordingly (e.g., adjusting blood pressure relative to moment-by-moment demands). For a much more detailed discussion, see Sterling, 2012.
} 
depending on the difference (prediction error) between predicted (demanded) and actual signal (Barrett \& Simmons, 2015).

Throughout the process of adjusting interoceptive predictions and bodily states, emotional contents may arise (Barrett, 2017; Seth, 2013a). Visceromotor cortices engender autonomic, hormonal and immunological predictions which underlie the system's allostatic responses. These predictions are not only forwarded to the body where they cause internal changes, they are also sent to the insula, termed the 'primary interoceptive cortex' (Barrett \& Simmons, 2015). Here these predictions are compared to afferent signals resulting from visceral, muscular and skin changes. The ensuing error signal is propagated back up to visceromotor regions where the prediction originated. Once interoceptive input is accounted for, that is, once it is sufficiently explained by predictive models, emotional content arises (Barrett, 2017).

This model of emotions draws on the James-Lange theory, which assumes that emotional experiences are first and foremost constituted by the monitoring of interoceptive states. However, one shortcoming of this theory, which has been adopted and refined widely (e.g. the somatic marker hypothesis, (Damasio, 1996), is that it (at least implicitly) asserts a one-to-one mapping of emotional effect and interoceptive cause (Clark, 2016). This means that it is assumed that there is one change in interoception (that can probably be marked, hence 'somatic marker hypothesis') that leads to the perceived emotion. However, it is more likely that there are several possible causes that bring forth an emotion. Viewing this basic idea within an IPP perspective, though, promises to alleviate this problem of former theories in the sense that the most probable of a variety of likely reasons is actively inferred.

IPP furthermore describes a strong relation between exteroceptive, proprioceptive, and interoceptive processes in that it assumes that information from all three channels is integrated at hierarchically higher levels. It is this "context-reflecting amalgam" (Clark, 2016) that then presents itself as an experienced emotion. Emotions, in this view, integrate lowlevel uni-modal information (i.e., interoceptive, exteroceptive, or proprioceptive information) with higher-level predictions of probable causes. In other words, emotions occur in the process of integrating information from several sources in order to form interoceptive predictions. These predictions, which then contain contextual information from exteroception, interoception, and proprioception are compared to actual incoming signals. Emotional states arise throughout this process. Note that this implies an intimate, and likely interacting relation between interoceptive and exteroceptive processing. For one, both external and internal events shape emotional response. Additionally, when external events trigger a cascade of interoceptive processing, these events will be "affectively coloured" (Clark, 2016). 
One important consequence of adopting such a view is that it updates appraisal or two-factor theories of emotional experience (Schachter \& Singer, 1962). These theories suggest that emotions have two components: a bodily and a cognitive one. The latter is needed to appraise the former and thus leaves the emotion subjectively colored. However, IPP now claims that subjective emotional states simply occur in virtue of the single mechanism just described. Multimodal higher-level top-down predictions entail contextual information which frame how interoceptive signals are interpreted and transformed into emotions. This means that in the process of interoceptive predictive processing, cognitive appraisal is already 'built in' and shapes emotional experience.

The role of the insular cortex, especially the AIC, undoubtedly plays a key role in this coordinating process. Seth (2013) claims that emotional responses crucially rely on predictions of causes of interoceptive input that are continually updated. These predictions are generated, compared to actual input and then updated within a salience network that consists of insular cortex, ACC and several other functional connections to the brainstem.

IPP is a promising theory to frame the relationship between emotion and interoception because it proposes a single principle- prediction error minimization - to underlie the generation of emotional and bodily states. It also provides novel ways to look at psychopathology, which we will detail in the next section.

\section{Clinical Implications}

Many psychiatric conditions come with emotion impairments - ranging from prevalent negative affect in depression to feelings of high alert and arousal in anxiety. A clear example of how emotion and interoception are related in psychopathology is the connection between interoceptive performance and occurrence of alexithymia. It has been proposed that where there is alexithymia, there is poor interoceptive accuracy (Murphy, Brewer, Catmur, \& Bird, 2016). Indeed, many clinical conditions come with both alexithymia and impairments in interoceptive processing. Here, we will focus on autism spectrum conditions (ASCs), anxiety disorders, depression and eating disorders (EDs), all of which show an interesting relation between emotional and interoceptive processing.

\section{Autism Spectrum Conditions}

Autism spectrum conditions are characterized as neurodevelopmental lifelong conditions that come with impairments in social cognition and emotion processing, as well as restrictions in interest and activities and stereotyped patterns of behaviour (Frith, 2014). ASCs express high rates of alexithymia (Bird, et al., 2010), which indicates difficulties in processing and detecting own emotional states. This impairment extends into the social realm where the detection of emotions in others can be exacerbated. 
A predictive processing perspective has been used to explain several aspects of the symptomology of ASCs, including unusual perception and sensation (Pellicano \& Burr, 2012) and impairments in social cognition (Palmer, Seth, \& Hohwy, 2015). Aiming at an overarching predictive processing account of autism, it was claimed that sensory processing in individuals with ASCs is characterized by an unusually high weighting of sensory prediction errors (Palmer, Lawson, \& Hohwy, 2017).

An aberrant oxytocin system in early infancy may play a role in the development of autistic traits (Quattrocki \& Friston, 2014). The neuromodulatory effects of oxytocin perform a major part in the processing of interoceptive information and their association with exteroceptive stimuli, and may also be involved in the neuromodulations that are necessary to elicit emotions. The functional anatomy of oxytocin is situated in the brain in such a way that it is involved in interoceptive paths that also mediate emotions. Oxytocin is thought to have an important role in the modulation of associative learning between interoceptive and exteroceptive cues. To learn emotional affordances (e.g., caregiver means warmth and comfort) during infancy, associations between exteroceptive cues (touch) and interoceptive signals (warmth) need to be established. Neuromodulatory mechanisms are involved in making these associations. For example, the interoceptive change of body temperature is associated with the bodily presence of a caregiver due to neuromodulatory mechanisms that underlie the establishment of this association. This is thought to be done via selective augmentation and attenuation of interoceptive prediction errors, likely mediated by oxytocin. If these mechanisms go awry, however, the context-sensitive adjustment of prediction errors is disrupted, resulting in a disturbance of the necessary mechanisms to acquire generative models for interoceptive inference which target the emotional states of self and other. In other words, the failure to associate the interoceptive consequence (warmth and comfort) with the exteroceptive cause (caregiver) may lead to an incorrect attribution of agency in prosocial actions. Impaired interoceptive inference, following this logic, could thus lead to difficulty in recognizing emotions in self and others. The failure to modulate precision of interoceptive prediction error may thus result in hypersensitivity to interoceptive cues ("autonomic hypersensitivity"). This is because when interoceptive signals are always weighted as precise, they will have an increased propensity to be propagated back up the hierarchy, and consequently, have the power to change predictions. This, in turn, could lead to difficulties in both emotional and interoceptive processing.

Viewing autism as a consequence of an abnormal neuromodulatory system (including oxytocin) provides an interesting perspective on well-known symptoms like emotional echopraxia (which can be interpreted as interoceptive hypersensitivity) and the tendency to not engage with exteroceptive cues in social settings. This explanation points to 
aberrant precision control in brain systems that mediate interoceptive inference, such as anterior insular and cingulate cortex (Seth \& Friston, 2016).

On a neural level, autism studies report altered activity in regions that underlie emotion processing, as well as impoverished functional connectivity in regions important to both interoception and emotion. Ebisch and colleagues (Ebisch et al., 2011) quantified aberrant functional connectivity between posterior insula and somatosensory cortices, as well as between anterior insula and amygdala. These regions are hubs for interoception and emotion (Critchley, Mathias, \& Dolan, 2002), suggesting not only a link between the two in healthy individuals, but also in individuals with ASCs. Based on this evidence, it was shown that impairments in interoceptive processing are related to aberrant emotional processing in ASCs (Garfinkel, et al., 2016). This appears to confirm theories of emotion which state that the detection of internal signals informs emotional experience, since it shows that when detection is disrupted, emotional impairments follow. Another indication of a relationship between aberrant interoception, emotion and ASCs is that these conditions often co-occur with heightened alexithymia. Interestingly it was shown, however, that levels of alexithymia and not ASC are predictive of low interoceptive accuracy (Shah, Hall, Catmur, \& Bird, 2016). Thus poor interoceptive accuracy may serve as a general feature of alexithymia, as well as underscore alexithymic traits in individuals with ASCs.

A discrepancy between levels of interoception, i.e., enhanced interoceptive sensibility and low interoceptive accuracy, is predictive of anxiety symptomology. This discrepancy was termed 'interoceptive trait prediction error' (ITPE) and was found to be enhanced in individuals with ASCs (Garfinkel et al., 2016). This finding is in line with the proposal that the development of anxiety is due to both noisy interoceptive input and noisily amplified selfreferential interoceptive belief states (Paulus \& Stein, 2010). Viewing the relation between anxiety and autism in terms of ITPE is based on the fine grained distinction of psychological dimensions of interoception. Where previous findings suggest that there is heightened sensitivity to bodily signals in autism, the differentiation between interoceptive sensibility and accuracy helps identify how interoceptive processing is altered autism.

The accounts of autism described here assign an important role of prediction error processing to symptoms of ASC. However, there are crucial differences between the types of prediction errors mentioned. While in general IPP, prediction errors refer to error signals occurring on a moment-to-moment basis in interoceptive processing, ITPE describes a relationship between subjective and objective traits. They thus characterize relevant error signals on different time scales, both of which could contribute to the occurrence of autistic characteristics. 
Taken together, these results suggest that alignment and misalignment of interoceptive dimensions are fundamental to affective symptoms in autism, and - as will be detailed in following sections - other clinical conditions.

\section{Anxiety Disorders}

The misalignment of interoceptive dimensions may be especially important for the development of anxiety disorders (ADs). The misattribution of bodily sensations is a factor underlying the development of panic and related anxiety symptoms (Clark et al., 1997). The basis for misinterpreted bodily signals forms a heightened tendency to detect internal physiological changes. Indeed, several studies showed that higher interoceptive accuracy is related to higher anxiety traits (Domschke, Stevens, Pfleiderer, \& Gerlach, 2010).

In a model of anxiety that focuses on the role of the insula in the processing of bodily signals, the ability to detect sub-threshold interoceptive signals is identified as one major mechanism in the expression of anxiety (Paulus \& Stein, 2006, 2010). These signals are then amplified and associated with potential aversive outcomes, thus forming the basis for anxious thought. Individuals with high anxiety traits are thought to be particularly sensitive to changes in interoceptive state when they anticipate ensuing aversive events. Belief-based thought with the tendency to focus on potential negative outcomes then strongly modifies the emotional valence of amplified bodily signals and leads to anxiety symptoms.

From an IPP perspective, it is likely that anxiety symptoms stem from abnormal precision weighting processes. In line with previous theories, aberrantly large interoceptive prediction errors lead to heightened anxiety. Whereas in healthy individuals these errors get attenuated, this mechanism might fail in anxious individuals, causing an abnormally high sensitivity to afferent interoceptive signals. In other words, bodily signals are weighted high much more often in anxious than healthy individuals. This means that these signals are more likely to be propagated back up the hierarchy and update predictions at higher levels. Where interoceptive input is usually attenuated and does not reach awareness, this mechanism might be impaired in anxiety, leading to a hypersensitivity to interoceptive signals. This is then also reflected in higher interoceptive accuracy, where bodily signals are more readily detected and available to conscious perception.

\section{Depression}

Major depressive disorder (MDD) is marked by a range of somatic and affective symptoms, including loss of appetite, sleep disorders, aches and pains, changes in libido, loss of energy, pervasive negative affect and intense feelings of loneliness and hopelessness (Tylee \& Gandhi, 2005). Somatic symptoms are universal cross-culturally (Kirmayer, 2001) and associated with disruptions in physiological regulatory processes, including hypoactivity of the autonomic nervous system (Dawson, Schell, \& Catania, 1977). 
Over the past decade, there has been growing recognition that somatic changes may drive alterations in emotion and cognition (Dunn, Dalgleish, Ogilvie, \& Lawrence, 2007; Pollatos, Traut-Mattausch, \& Schandry, 2009). The role of interoception in low mood and clinical depression has been investigated in a number of studies; interoceptive accuracy is reduced in low to moderate, but not severe depression (Dunn, et al., 2007). A negative correlation between heartbeat perception and depressive symptoms are reported (Pollatos, et al., 2009), where individuals with reduced interoceptive accuracy may experience emotions less intensely. These emotional impairments will impact social processes, as is often observed in MDD.

There is frequent co-morbidity of depression and anxiety, possibly confounding interpretations. One subsequent study chose depressed subjects with no co-morbid anxiety disorder (Furman, Waugh, Bhattacharjee, Thompson, \& Gotlib, 2013). An association between interoceptive accuracy and positive affect intensity was observed. Moreover worse performance was related to less affect intensity ratings in individuals with MDD, but not controls. The reduced autonomic reactivity to positive stimuli in depression (Bylsma, Morris, \& Rottenberg, 2008), and the association between depressive symptoms and decreased responses to positive stimuli (Sloan \& Sandt, 2010), indicate an aberrant interoceptive mechanism for positive reinforcement. In other words, physiological disruption in depression limits the intensity of interoceptive input. This in turn results in attenuated affective reactions to positive information.

Depression was recently framed in the PP framework and described as a disorder of predictive mechanisms that steer allostasis, i.e., the ability to flexibly adapt to changing physiologically internal and external environments (Barrett, Quigley, \& Hamilton, 2016). Affective feelings can inform the system about the state of bodily energy conditions, whereby the inefficient regulation of energy is coupled to affective, motor, autonomic and metabolic dysregulations. In depression, the brain's internal model is negatively affected by this dysregulation. The brain is described as 'locked in' in the sense that there is an insensitivity to interoceptive prediction errors which - coupled with energy dysregulation that is related to negative affect - leads to depression. In more detail, this means that when interoceptive prediction errors (i.e., bodily signals) are not used to update predictions, these predictions may result in inefficient energy regulation. This inefficiency could be related to negative affect, and the tendency to disengage in physical and mental activity. Pervasive negative affect, in turn, could lead to the generation of more and more negative internal models, resulting in both predictions and prediction errors to show a bias towards the negative and unpleasant.

Furthermore, peripheral endocrine immunological changes that accompany the onset of a depressive episode can perturb precision of interoceptive afferents (Seth \& Friston, 
2016). The attenuation of precision of ascending interoceptive signals, and greater reliance on interoceptive predictions, lead to dyshomeostasis. This process could engender a positive feedback loop in which higher reliance on predictions leads to larger and nosier prediction errors, which in turn increases the reliance on the now dysfunctional predictions. Since interoceptive predictions include 'homeostatic set-points', dysfunctional prediction will result in dysregulated homeostasis, which may trigger sickness behaviour and fatigue, marking the onset of depression.

Taken together these findings suggest that dysfunctions in interoception and autonomic regulation are strongly related to both somatic and emotional symptoms of depression. PP models of depression exhibit a strong connection between allostasis/homeostasis and the resulting dysbalance of affective processing.

\section{Eating Disorders}

Both interoceptive and emotional processing appear impaired in eating disorders. Alexithymia often co-occurs with EDs, indicating a generally impaired recognition of own emotions. This extends into the social realm where it was found that emotion recognition in others is diminished in patients with eating disorders (Harrison, Sullivan, Tchanturia, \& Treasure, 2009). Emotion recognition performance, however, was predicted by alexithymia scores rather than eating disorder symptomology (Brewer, Cook, Cardi, Treasure, \& Bird, 2015). What still stands out, though, is the high co-occurrence of alexithymia and EDs, suggesting that emotional processing is indeed impaired in eating disorders.

When investigated directly, there appears to be a three-way relation between interoceptive performance, emotional intensity and symptomology of eating disorders. So far, interoceptive abilities in EDs were tested by the Eating Disorder Inventory (EDI; Garner, Olmstead \& Polivy, 1983), in which the ability to discriminate sensations of hunger and satiety and to respond to emotional states ("interoceptive awareness") ${ }^{3}$ was in focus. Interoceptive awareness, as measured by the EDI, was impaired in patients with eating disorders, where this is characterized as a low certainty in emotion recognition related to hunger and satiety (Fassino, Pierò, Gramaglia, \& Abbate-Daga, 2004).

When using the heartbeat counting task (Schandry, 1981) to quantify interoceptive accuracy, decreased measures were found in patients with eating disorders compared to healthy controls (Pollatos et al., 2008). This shows that interoceptive ability is impaired in general and not just with respect to hunger and satiety, as measured by the EDI.

\footnotetext{
${ }^{3}$ Note how the term "interoceptive awareness" here relates to the specific measure of discrimination of sensations and response to emotional states and is different from how we use the term in previous sections as a measure of the relationship between objective and subjective scores.
} 
Together with findings that show altered autonomic functioning in eating disorders (Murialdo et al., 2007) which could lead to altered bodily feedback, this suggests that individuals with EDs experience emotions less intensely. Evidence in favor of this claim comes again from studies linking alexithymia to eating disorders (Berthoz, Perdereau, Godart, Corcos, \& Haviland, 2007) and showing diminished affect in patients (de Zwaan, Biener, Bach, Wiesnagrotzki, \& Stacher, 1996).

Eating disorders could be a result of aberrant precision weighting when viewed from an IPP perspective (Seth, 2015). In PP, only prediction errors that are deemed 'reliable' will be propagated back up the hierarchy and update predictions. This means that in healthy individuals, interoceptive prediction errors related to blood sugar levels are generally weighted high in order to update predictions and lead to the sensation of hunger when the levels drop below a specific threshold. The desired, predicted bodily state thus is different from the actual state, which leads to the generation of interoceptive prediction errors. These in turn are forwarded to higher-level, multimodal models. Such models generate predictions of temporal sequences of exteroceptive and interoceptive inputs which are propagated down the hierarchy. The ensuing prediction errors can then be resolved by either autonomic control, where fat stores are metabolized, or by engaging active movements to seek food resources. It is hypothesized that dysfunctions in precision weighting, which steers this process, may help understanding EDs. If, for example, it is constantly assumed that blood sugar will be low (i.e., high-level predictions of low blood sugar become aberrantly strong), behaviour to find food may not pursue.

Taken together, these findings suggest that problems in the discrimination of sensations like hunger and satiety, which are deemed at the core of the onset and maintenance of eating disorders, are mediated by a more general inability to regulate and describe emotions. This in turn can be traced back to impairments in interoceptive ability, showing a link between interoception and emotion in eating disorders.

\section{Conclusion}

Links between interoception and emotion can be found in the relationship between interoceptive performance and experience of emotional intensity, the ability to recognize one's own emotions (as measured in alexithymia scores) and interoceptive accuracy, and emotion and interoception in psychopathology.

The framework of IPP gives us a means to put these findings together in terms of interoceptive inference and integration of multimodal information to generate interoceptive predictions. Aberrant precision weighting may be involved in psychopathology, as was hypothesized for depression and eating disorders. 
Together, experimental and clinical findings support the proposal that interoception, including individual differences in interoceptive processing, guides the experience and expression of emotions. Altered interoceptive pathways may account for aberrant emotional processing in psychopathological disorders. These observations provide insights into potential body-centered therapies and pharmacological targets for novel interventions for clinical disorders of emotion. Where interoceptive dimensions diverge, leading to psychopathological symptoms like anxiety, training interoceptive accuracy could lead to a reduction in these symptoms. Thus insight into specific interoceptive deficits may offer a targeted therapeutic approach for clinical conditions with altered emotional and affective processing.

\section{References}

Ainley, V., Maister, L., \& Tsakiris, M. (2015). Heartfelt empathy? No association between interoceptive awareness, questionnaire measures of empathy, reading the mind in the eyes task or the director task. Frontiers in Psychology, 6, 554. doi: 10.3389/fpsyg.2015.00554

Barrett, L. F. (2017). The theory of constructed emotion. Social Cognitive and Affective Neuroscience, 1-13.

Barrett, L. F., Quigley, K. S., \& Hamilton, P. (2016). An active inference theory of allostasis and interoception in depression. Philosophical Transaction of the Royal Society B, 371(1708), 20160011.

Barrett, L. F., \& Simmons, W. K. (2015). Interoceptive predictions in the brain. Nat Rev Neurosci, 16(7), 419.

Berthoz, S., Artiges, E., Van De Moortele, P. F., Poline, J. B., Rouquette, S., Consoli, S. M., \& Martinot, J. L. (2002). Effect of impaired recognition and expression of emotions on frontocingulate cortices: an fMRI study of men with alexithymia. The American Journal of Psychiatry, 159(6), 961-967. doi: 10.1176/appi.ajp.159.6.961

Berthoz, S., Perdereau, F., Godart, N., Corcos, M., \& Haviland, M. G. (2007). Observer-and self-rated alexithymia in eating disorder patients: Levels and correspondence among three measures. Journal of Psychosomatic Research, 62(3), 341-347.

Bird, G., Silani, G., Brindley, R., White, S., Frith, U., \& Singer, T. (2010). Empathic brain responses in insula are modulated by levels of alexithymia but not autism. Brain, 133(Pt 5), 1515-1525. doi: 10.1093/brain/awq060

Brewer, R., Cook, R., Cardi, V., Treasure, J., \& Bird, G. (2015). Emotion recognition deficits in eating disorders are explained by co-occurring alexithymia. Royal Society open science, 2(1), 140382.

Bylsma, L. M., Morris, B. H., \& Rottenberg, J. (2008). A meta-analysis of emotional reactivity in major depressive disorder. Clinical Psychology Review, 28(4), 676-691.

Canales-Johnson, A., Silva, C., Huepe, D., Rivera-Rei, Á., Noreika, V., del Carmen Garcia, M., . . . Sedeño, L. (2015). Auditory feedback differentially modulates behavioral and neural markers of objective and subjective performance when tapping to your heartbeat. Cerebral Cortex, bhv076. 
Clark, A. (2013). Whatever next? Predictive brains, situated agents, and the future of cognitive science. Behavioral Brain Sciences, 36(3), 181-204. doi: 10.1017/S0140525X12000477

Clark, A. (2015). Embodied Prediction. In T. K. Metzinger \& J. M. Windt (Eds.), Open MIND. Frankfurt am Main: MIND Group.

Clark, A. (2016). Surfing Uncertainty: Prediction, Action and the Embodied Mind. Oxford: Oxford University Press.

Clark, D. M., Salkovskis, P. M., Öst, L.-G., Breitholtz, E., Koehler, K. A., Westling, B. E., . . Gelder, M. (1997). Misinterpretation of body sensations in panic disorder. Journal of consulting and clinical psychology, 65(2), 203.

Craig, A. D. (2002). How do you feel? Interoception: the sense of the physiological condition of the body. Nature Reviews Neuroscience, 3(8), 655-666. doi: 10.1038/nrn894

Critchley, H. D. (2004). The human cortex responds to an interoceptive challenge. Proc Natl Acad Sci U S A, 101(17), 6333-6334. doi: 10.1073/pnas.0401510101

Critchley, H. D., Corfield, D., Chandler, M., Mathias, C., \& Dolan, R. J. (2000). Cerebral correlates of autonomic cardiovascular arousal: a functional neuroimaging investigation in humans. The Journal of physiology, 523(1), 259-270.

Critchley, H. D., \& Garfinkel, S. N. (2015). Interactions between visceral afferent signaling and stimulus processing. Front Neurosci, 9, 286. doi: 10.3389/fnins.2015.00286

Critchley, H. D., \& Garfinkel, S. N. (2017). Interoception and emotion. Current Opinion in Psychology, 17, 7-14. doi: https://doi.org/10.1016/j.copsyc.2017.04.020

Critchley, H.D., \& Harrison, N.A. (2013). Visceral Influences on Brain and Behavior. Neuron, 77(4) :624-38. doi: 10.1016/j.neuron.2013.02.008.

Critchley, H. D., Mathias, C. J., \& Dolan, R. J. (2002). Fear conditioning in humans: the influence of awareness and autonomic arousal on functional neuroanatomy. Neuron, 33(4), 653-663.

Critchley, H. D., Rotshtein, P., Nagai, Y., O'Doherty, J., Mathias, C. J., \& Dolan, R. J. (2005). Activity in the human brain predicting differential heart rate responses to emotional facial expressions. Neuroimage, 24(3), 751-762. doi: 10.1016/j.neuroimage.2004.10.013

Critchley, H. D., Wiens, S., Rotshtein, P., Ohman, A., \& Dolan, R. (2004). Neural systems supporting interoceptive awareness. Nature Neuroscience, 7(2), 189-195. doi: 10.1038/nn1176

Damasio, A. R. (1996). The somatic marker hypothesis and the possible functions of the prefrontal cortex. Philosophical Transactions Royal Society London B Biological Sciences, 351(1346), 1413-1420. doi: 10.1098/rstb.1996.0125

Dawson, M. E., Schell, A. M., \& Catania, J. J. (1977). Autonomic correlates of depression and clinical improvement following electroconvulsive shock therapy. Psychophysiology, 14(6), 569-578.

de Zwaan, M., Biener, D., Bach, M., Wiesnagrotzki, S., \& Stacher, G. (1996). Pain sensitivity, alexithymia, and depression in patients with eating disorders: are they related? Journal of Psychosomatic Research, 41(1), 65-70.

Deen, B., Pitskel, N. B., \& Pelphrey, K. A. (2011). Three Systems of Insular Functional Connectivity Identified with Cluster Analysis. Cerebral Cortex, 21(7), 1498-1506. doi: 10.1093/cercor/bhq186

Domschke, K., Stevens, S., Pfleiderer, B., \& Gerlach, A. L. (2010). Interoceptive sensitivity in anxiety and anxiety disorders: an overview and integration of neurobiological findings. Clinical Psychology Review, 30(1), 1-11. doi: 10.1016/j.cpr.2009.08.008

Dunn, B. D., Dalgleish, T., Ogilvie, A. D., \& Lawrence, A. D. (2007). Heartbeat perception in depression. Behavior Research and Therapy, 45(8), 1921-1930.

Ebisch, S. J., Gallese, V., Willems, R. M., Mantini, D., Groen, W. B., Romani, G. L., . . Bekkering, H. (2011). Altered intrinsic functional connectivity of anterior and posterior insula regions in high-functioning participants with autism spectrum disorder. Human Brain Mapping, 32(7), 1013-1028. doi: 10.1002/hbm.21085

Ernst, J., Böker, H., Hättenschwiler, J., Schüpbach, D., Northoff, G., Seifritz, E., \& Grimm, S. (2014). The association of interoceptive awareness and alexithymia with neurotransmitter 
concentrations in insula and anterior cingulate. Social Cognitive and Affective Neuroscience, 9(6), 857-863. doi: 10.1093/scan/nst058

Ernst, J., Northoff, G., Böker, H., Seifritz, E., \& Grimm, S. (2013). Interoceptive awareness enhances neural activity during empathy. Human brain mapping, 34(7), 1615-1624.

Fassino, S., Pierò, A., Gramaglia, C., \& Abbate-Daga, G. (2004). Clinical, psychopathological and personality correlates of interoceptive awareness in anorexia nervosa, bulimia nervosa and obesity. Psychopathology, 37(4), 168-174.

Friston, K. (2010). The free-energy principle: a unified brain theory? [10.1038/nrn2787]. Nature Review Neuroscience, 11(2), 127-138. doi: http://www.nature.com/nrn/journal/v11/n2/suppinfo/nrn2787 S1.html

Frith, U. (2014). Autism-are we any closer to explaining the enigma? Psychologist, 27(10), 744-745.

Furman, D. J., Waugh, C. E., Bhattacharjee, K., Thompson, R. J., \& Gotlib, I. H. (2013). Interoceptive awareness, positive affect, and decision making in major depressive disorder. Journal of affective disorders, 151(2), 780-785.

Garfinkel, S. N., \& Critchley, H. D. (2016). Threat and the Body: How the Heart Supports Fear Processing. Trends Cogn Sci, 20(1), 34-46. doi: 10.1016/j.tics.2015.10.005

Garfinkel, S. N., Minati, L., Gray, M. A., Seth, A. K., Dolan, R. J., \& Critchley, H. D. (2014). Fear from the heart: sensitivity to fear stimuli depends on individual heartbeats. J Neurosci, 34(19), 6573-6582. doi: 10.1523/JNEUROSCI.3507-13.2014

Garfinkel, S. N., Seth, A. K., Barrett, A. B., Suzuki, K., \& Critchley, H. D. (2015). Knowing your own heart: distinguishing interoceptive accuracy from interoceptive awareness. Biological Psychology, 104, 65-74. doi: 10.1016/j.biopsycho.2014.11.004

Garfinkel, S. N., Tiley, C., O'Keeffe, S., Harrison, N. A., Seth, A. K., \& Critchley, H. D. (2016). Discrepancies between dimensions of interoception in autism: Implications for emotion and anxiety. Biological Psychology, 114, 117-126. doi: 10.1016/j.biopsycho.2015.12.003

Garner, D. M., Olmstead, M. P., \& Polivy, J. (1983). Development and validation of a multidimensional eating disorder inventory for anorexia nervosa and bulimia. International journal of eating disorders, 2(2), 15-34.

Harrison, A., Sullivan, S., Tchanturia, K., \& Treasure, J. (2009). Emotion recognition and regulation in anorexia nervosa. Clinical psychology \& psychotherapy, 16(4), 348-356.

Harrison, N. A. (2017). Brain Structures Implicated in Inflammation-Associated Depression. In R. Dantzer \& L. Capuron (Eds.), Inflammation-Associated Depression: Evidence, Mechanisms and Implications (pp. 221-248). Cham: Springer International Publishing.

Herbert, B. M., Pollatos, O., \& Schandry, R. (2007). Interoceptive sensitivity and emotion processing: an EEG study. International Journal of Psychophysiology, 65(3), 214-227.

Hohwy, J. (2010). The hypothesis testing brain: some philosophical applications.

Hohwy, J. (2013). The predictive mind: Oxford University Press.

James, W. (1884). What is an emotion? Mind, 9, 188-205.

Katkin, E. S., Morell, M. A., Goldband, S., Bernstein, G. L., \& Wise, J. A. (1982). Individual differences in heartbeat discrimination. Psychophysiology, 19(2), 160-166.

Kirmayer, L. J. (2001). Cultural variations in the clinical presentation of depression and anxiety: implications for diagnosis and treatment. Journal of Clinical Psychiatry, 62, 22-30.

Kober, H., Barrett, L. F., Joseph, J., Bliss-Moreau, E., Lindquist, K., \& Wager, T. D. (2008). Functional grouping and cortical-subcortical interactions in emotion: a meta-analysis of neuroimaging studies. Neuroimage, 42(2), 998-1031. doi: 10.1016/j.neuroimage.2008.03.059

Lamm, C., \& Singer, T. (2010). The role of anterior insular cortex in social emotions. Brain Struct Funct, 214(5-6), 579-591. doi: 10.1007/s00429-010-0251-3

Murialdo, G., Casu, M., Falchero, M., Brugnolo, A., Patrone, V., Cerro, P., . . . Copello, F. (2007). Alterations in the autonomic control of heart rate variability in patients with anorexia or bulimia nervosa: correlations between sympathovagal activity, clinical features, and leptin levels. Journal of endocrinological investigation, 30(5), 356-362. 
Murphy, J., Brewer, R., Catmur, C., \& Bird, G. (2016). Interoception and psychopathology: A developmental neuroscience perspective. Developmental Cognitive Neuroscience.

Nemiah, J. C. (1976). Alexithymia: a view of the psychosomatic process. Modern trends in psychosoamtic medicine, 3, 430-439.

Nummenmaa, L., Glerean, E., Hari, R., \& Hietanen, J. K. (2014). Bodily maps of emotions. Proc Natl Acad Sci U S A, 111(2), 646-651. doi: 10.1073/pnas.1321664111

Palmer, C. J., Lawson, R. P., \& Hohwy, J. (2017). Bayesian approaches to autism: Towards volatility, action, and behavior. Psychol Bull, 143(5), 521-542. doi: 10.1037/bul0000097

Palmer, C. J., Seth, A. K., \& Hohwy, J. (2015). The felt presence of other minds: Predictive processing, counterfactual predictions, and mentalising in autism. Conscious Cogn, 36, 376-389. doi: 10.1016/j.concog.2015.04.007

Paulus, M. P., \& Stein, M. B. (2006). An insular view of anxiety. Biol Psychiatry, 60(4), 383-387. doi: 10.1016/j.biopsych.2006.03.042

Paulus, M. P., \& Stein, M. B. (2010). Interoception in anxiety and depression. Brain Structure and Function, 214(5-6), 451-463. doi: 10.1007/s00429-010-0258-9

Pellicano, E., \& Burr, D. (2012). When the world becomes 'too real': a Bayesian explanation of autistic perception. Trends in cognitive sciences, 16(10), 504-510.

Peyron, R., Laurent, B., \& Garcia-Larrea, L. (2000). Functional imaging of brain responses to pain. A review and meta-analysis (2000). Neurophysiol Clin, 30(5), 263-288.

Phan, K. L., Wager, T., Taylor, S. F., \& Liberzon, I. (2002). Functional neuroanatomy of emotion: a meta-analysis of emotion activation studies in PET and fMRI. Neuroimage, 16(2), 331-348.

Pollatos, O., Kurz, A.-L., Albrecht, J., Schreder, T., Kleemann, A. M., Schöpf, V., . . S Schandry, R. (2008). Reduced perception of bodily signals in anorexia nervosa. Eating behaviors, 9(4), 381-388.

Pollatos, O., Traut - Mattausch, E., \& Schandry, R. (2009). Differential effects of anxiety and depression on interoceptive accuracy. Depress Anxiety, 26(2), 167-173.

Porges, S. (1993). Body perception questionnaire. Laboratory of Developmental Assessment, University of Maryland.

Quadt, L. (2017). Levels of social embodiment - towards a unifying perspective on social cognition. Mainz.

Quattrocki, E., \& Friston, K. (2014). Autism, oxytocin and interoception. Neurosci Biobehav Rev, 47, 410-430. doi: 10.1016/j.neubiorev.2014.09.012

Schachter, S., \& Singer, J. E. (1962). Cognitive, social, and physiological determinants of emotional state. Psychological Review, 69, 379-399.

Schandry, R. (1981). Heart beat perception and emotional experience. Psychophysiology, 18(4), 483488.

Seth, A. K. (2013a). Interoceptive inference, emotion, and the embodied self. Trends in Cognitive Sciences, 17(11), 565-573. doi: 10.1016/j.tics.2013.09.007

Seth, A. K. (2013b). Interoceptive inference, emotion, and the embodied self. Trends Cogn Sci, 17(11), 565-573. doi: 10.1016/j.tics.2013.09.007

Seth, A. K. (2015). The Cybernetic Bayesian Brain. In T. K. Metzinger \& J. M. Windt (Eds.), Open MIND. Frankfurt am Main: MIND Group.

Seth, A. K., \& Friston, K. J. (2016). Active interoceptive inference and the emotional brain. Philosophical Transaction of the Royal Society B Biological Sciences, 371(1708). doi: 10.1098/rstb.2016.0007

Seth, A. K., Suzuki, K., \& Critchley, H. D. (2011). An interoceptive predictive coding model of conscious presence. Front Psychol, 2, 395. doi: 10.3389/fpsyg.2011.00395

Shah, P., Hall, R., Catmur, C., \& Bird, G. (2016). Alexithymia, not autism, is associated with impaired interoception. Cortex, 81, 215-220. 
Silani, G., Bird, G., Brindley, R., Singer, T., Frith, C., \& Frith, U. (2008). Levels of emotional awareness and autism: an fMRI study. Social Neuroscience, 3(2), 97-112. doi: 10.1080/17470910701577020

Singer, T., Critchley, H. D., \& Preuschoff, K. (2009). A common role of insula in feelings, empathy and uncertainty. Trends Cogn Sci, 13(8), 334-340. doi: 10.1016/j.tics.2009.05.001

Sloan, D. M., \& Sandt, A. R. (2010). Depressed mood and emotional responding. Biological Psychology, 84(2), 368-374.

Sterling, P. (2012). Allostasis: a model of predictive regulation. Physiol Behav, 106(1), 5-15.

Terasawa, Y., Moriguchi, Y., Tochizawa, S., \& Umeda, S. (2014). Interoceptive sensitivity predicts sensitivity to the emotions of others. Cognition and Emotion, 28(8), 1435-1448. doi: 10.1080/02699931.2014.888988

Terasawa, Y., Shibata, M., Moriguchi, Y., \& Umeda, S. (2013). Anterior insular cortex mediates bodily sensibility and social anxiety. Social Cognitive and Affective Neuroscience, 8(3), 259-266. doi: $10.1093 /$ scan/nss108

Tylee, A., \& Gandhi, P. (2005). The importance of somatic symptoms in depression in primary care. Primary Care Companion Journal of Clinical Psychiatry, 7(4), 167-176.

Wicker, B., Keysers, C., Plailly, J., Royet, J. P., Gallese, V., \& Rizzolatti, G. (2003). Both of us disgusted in My insula: the common neural basis of seeing and feeling disgust. Neuron, 40(3), 655-664.

Wiens, S., Mezzacappa, E. S., \& Katkin, E. S. (2000). Heartbeat detection and the experience of emotions. Cognition and Emotion, 14(3), 417-427. doi: 10.1080/026999300378905

Williams, L. M., Brammer, M. J., Skerrett, D., Lagopolous, J., Rennie, C., Kozek, K., . . . Gordon, E. (2000). The neural correlates of orienting: an integration of $\mathrm{FMRI}$ and skin conductance orienting. Neuroreport, 11(13), 3011-3015.

Zaki, J., Davis, J. I., \& Ochsner, K. N. (2012). Overlapping activity in anterior insula during interoception and emotional experience. Neuroimage, 62(1), 493-499. doi: 10.1016/j.neuroimage.2012.05.012 